\title{
Return to Work after Primary Total Knee Arthroplasty under the Age of 65 Years: A Systematic Review
}

\author{
Dries Van Leemput, $\mathrm{MD}^{1}$ (1) Jef Neirynck, $\mathrm{MD}^{1}{ }^{10}$ \\ ${ }^{1}$ Division of Orthopaedics, University Hospitals Leuven, B-3000 \\ Leuven, Belgium \\ 2 Division of Orthopaedics, Department of Development and \\ Regeneration-Organ Systems Cluster, KU Leuven, University \\ Hospitals Leuven, Belgium, B-3000 Leuven, Belgium
}

J Knee Surg 2022;35:1249-1259.

\author{
Pieter Berger, $\mathrm{MD}^{1}$ Hilde Vandenneucker, MD, $\mathrm{PhD}^{2}$
}

\begin{abstract}
Keywords

- work

- employment

- sick leave

- arthroplasty

- replacement

- knee
\end{abstract}

\author{
Address for correspondence Dries Van Leemput, MD, Division of \\ Orthopaedics, University Hospitals Leuven, B-3000 Leuven, Belgium \\ (e-mail: driesvanleemput@hotmail.com).
}

Osteoarthritis (OA) is one of the most common diseases of the knee, causing pain and often leading to disability and sick leave. Painful, severely disabling OA of the knee affects approximately $1.5 \%$ of adults over the age of 55 years. ${ }^{1}$

Total knee arthroplasty (TKA) is an effective treatment for patients with $\mathrm{OA}$, reducing pain and improving function and quality of life. ${ }^{2}$ This intervention has shown to have a good satisfaction rate, especially in older patients. ${ }^{3}$ However, in recent years, a growing portion of patients is undergoing TKA at working age and this is expected to rise even further in the

received

June 4, 2020

accepted after revision

November 29, 2020

published online

January 20, 2021 next years. ${ }^{4,5}$ Also, the average retirement age has increased to an average of 65 years in Europe and is expected to rise to 67 years in the next 10 years. $^{6}$ Therefore, an increasing number of knee OA patients will need to be able to return to work (RTW) after TKA. A previous systematic review showed that the proportions of RTW patients are ranging from 71 to $83 \%$ at 3 to 6 months after TKA. ${ }^{7}$

It is important to have insight in the rate of RTW for both individual and socioeconomic interests. Knowledge of determinants associated with a delayed return or no RTW could help (c) 2021. Thieme. All rights reserved. Thieme Medical Publishers, Inc., 333 Seventh Avenue, 18th Floor, New York, NY 10001, USA
DOI https://doi.org/ 10.1055/s-0040-1722626. ISSN 1538-8506. 
to focus on the right subpopulation of patients when applying appropriate management and preventive measures.

Systematic literature on this subject is scarce and outdated. The most recent systematic review, publicized in 2014, concluded that the literature on work status after total hip arthroplasty (THA) is more extensive than for TKA, as they only had four studies providing data on RTW after TKA. ${ }^{7}$ In addition, RTW after TKA seems to be a field of growing interest, with several studies being published in recent years. Therefore, a systematic summarization of the recent literature would be very useful.

The aim of this study is to provide an overview of the available literature on work status and RTW in patients under the age of 65 years undergoing primary TKA, and to determine beneficial and limiting factors associated with RTW postoperatively.

\section{Methods}

The selection process was conducted by two different authors independently (D.V.L and J.N). Any discrepancies in study selection were discussed until consensus was reached.

\section{Database Search}

A systematic database search was conducted in PubMed, Embase, Cochrane library, and Web of Science on November 18,2019 . A search strategy was developed for each database according to their specific search methods. The search strategy for PubMed consisted of the Medical Subject Headings (MeSH) terms "Return to work," "Sick Leave," and "Arthroplasty, Replacement, Knee." These terms were combined with synonyms in free words to obtain articles not covered by MeSH terms. The search strategy for each database can be found in the Supplementary data (available online only).

\section{Study Selection}

After removing the duplicates and the articles publicized before January 1, 2020, all titles and abstracts were screened. Articles were included for further selection if they met the following inclusion criteria: (1) patients underwent primary TKA, (2) articles reported on RTW after surgery, and (3) patients aged 65 years or below.

Next, using these same criteria, full-text articles were screened. Articles including revision TKA or hemiarthroplasty were excluded, unless they reported data for primary TKA separately. We included articles regardless of the indication for primary TKA. Articles investigating RTW after both TKA and THA were included if data were available for TKA separately.

\section{Data Extraction}

Study characteristics extracted were first author, year of publication, country, study type (retrospective or prospective), data collection method, indication for TKA, population size undergoing primary TKA, mean age, male/female ratio, and follow-up time.

Data extracted on work status were work status before surgery, description of work status after surgery, fraction of patients returning to work after surgery, time after surgery that RTW was measured, mean time taken to RTW, and influencing factors associated with RTW.

\section{Methodological Quality}

We assessed the risk for potential bias in included articles using the Quality in Prognosis Studies (QUIPS) checklist. This is a quality assessment tool developed to assess risk of bias in prognostic factor studies. It is based on a review discussing quality of prognostic studies in systematic reviews. ${ }^{8}$ This checklist consists of six domains being "study participation," "study attrition," "prognostic factor measurement," "outcome measurement," "study confounding," and "statistical analysis and reporting." Risk of bias was rated as high, moderate, or low depending on how many criteria in each domain were met. Two authors independently assessed the risk of bias in each study using this tool.

\section{Results}

\section{Search Results}

The initial search strategy provided 378 articles of which 292 remained after removing the duplicates. Thirteen articles published before the year 2000 were removed. After selection based on title and abstract, 53 articles remained for further analysis. Full-text screening of the selected articles resulted in 14 studies that met our eligibility criteria which were included in this review. A flow diagram of the selection process is shown in -Fig. 1.

\section{Study Characteristics}

Study characteristics of the included articles are presented in - Table 1. Articles were published between 2009 and 2019. Four studies had a prospective observational design and 10 studies had a retrospective design. Five studies were conducted in the United Kingdom, three in the Netherlands, two in the United States, one in Sweden, one in Canada, one in Finland, and one in Australia. The population sizes of the studies varied from six patients to 996 patients, and the articles together accounted for a total study population of 3,073 patients. The mean age ranged from 46 to 59 years. The indications for TKA were primarily OA. Two articles were qualitative studies using convenience sampling and with total population sizes of 6 and 10 patients, respectively. ${ }^{9,10}$ Therefore, we did not include RTW rates extracted from these two studies, as results would not be representative for the population. We did, however, include the patients' experiences and influencing factors reported in these studies.

\section{Return to Work}

- Table 2 shows the pre- and postoperative work status and the mean time taken to RTW. Work status prior to TKA was described in all studies, but the definition that was used differed among studies. Some articles reported the number of patients on sick leave in the months before surgery, others reported the number of patients in employment. Overall, the portion of patients working preoperatively ranged from 50 to $100 \%$. Four studies included only patients who were working before surgery. ${ }^{10-13}$ 


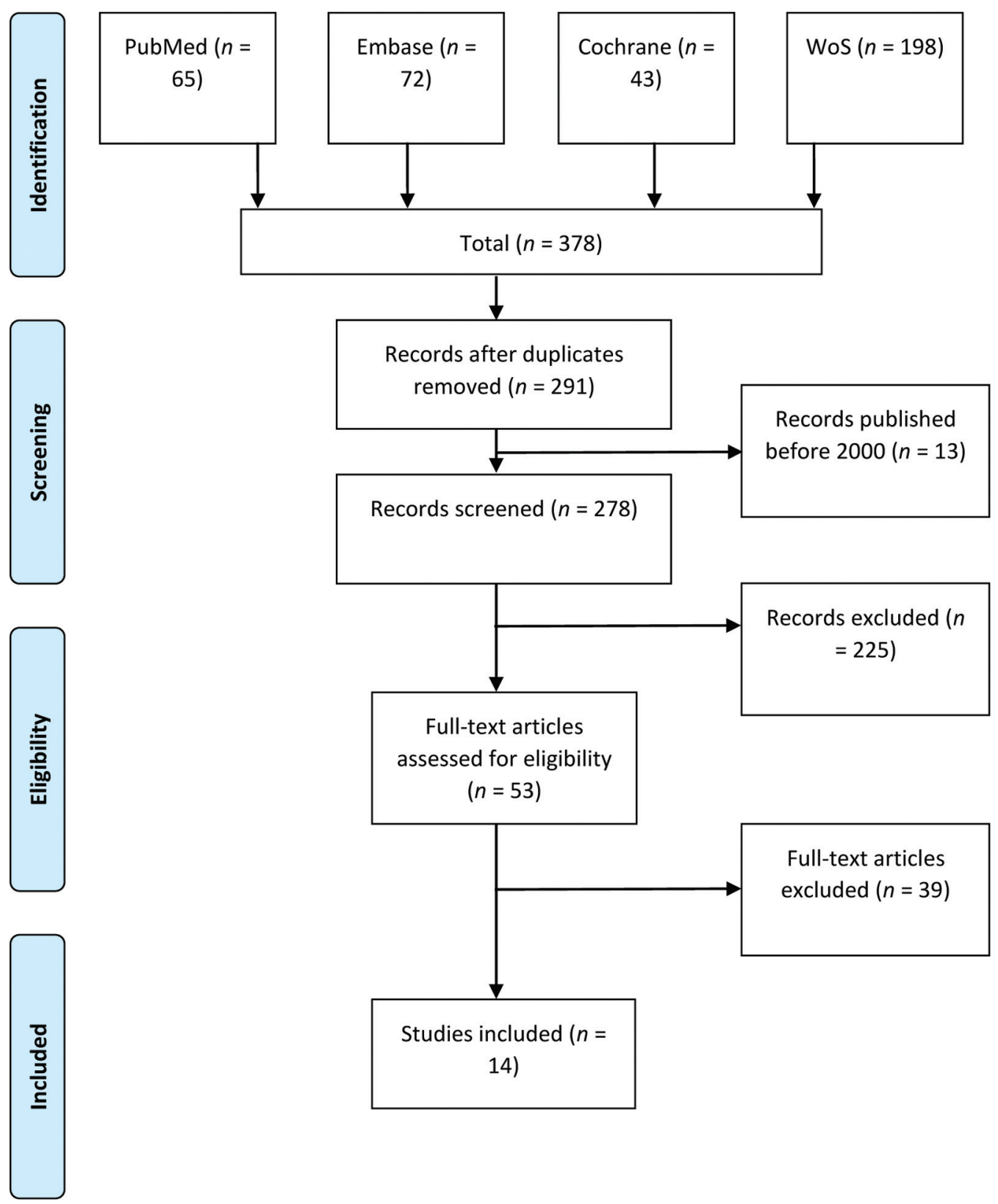

Fig. 1 Flowchart of the selection process. WoS, Web of Science.

All articles provided information on RTW after surgery. However, there was great variability concerning both the definition of work status postoperatively, as well as time after surgery when RTW was measured. The portion of patients working after TKA ranged from 36 to $95 \%$ (mean, $82 \%$ ), and the fraction of patients working before and returning to work after surgery ranged from 40 to $98 \%$ (mean, $89 \%$ ). Mean time taken to RTW ranged from 7.7 to 16.6 weeks (mean, 13 weeks).

Three articles specified whether patients returned to the same job or to a different job. In these three articles, 85, 91, and $100 \%$ of the RTW patients were returned to the same job. ${ }^{14-16}$ One article reported RTW rates for men and women separately. ${ }^{17}$ In this study, more men returned to work than women (men, 88\%; women, $83 \%$ ). No consistent differences in RTW rate were found between studies performed in different countries.

\section{Associated Factors}

An overview of the associated factors is shown in - Table 3. All studies mentioned factors that could be influencing RTW. Ten studies performed statistical analysis to investigate relations between RTW and variables. Seven of them did find one or more correlations to be statistically significant, and three studies performed statistical analysis but did not find any significant correlations. Six studies found significant correlation while applying multivariate analysis. ${ }^{11-14,18,19}$

Four studies did not perform any statistics but mentioned preferences or influencing factors reported by patients to be influencing their RTW. ${ }^{9}, 10,16,17$

Factors shown to be correlated with RTW by multivariate analysis were the physical nature of employment $\left(p=0.03,{ }^{14}\right.$ $0.012,{ }^{11}$ and $\left.<0.0001^{12,18}\right)$, preoperative absence from work $\left(p<0.0001^{18}\right.$ and $\left.0.001^{19}\right)$, flexible work conditions 


\begin{tabular}{|c|c|c|c|c|c|c|c|c|c|c|c|c|c|c|}
\hline 岕 & $=$ & 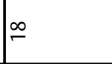 & $\simeq$ & $=$ & $\sigma$ & \pm & $\cong$ & $\cong$ & $\cong$ & $\curvearrowright$ & $\bar{N}$ & 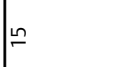 & i & $\cong$ \\
\hline 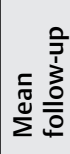 & 1 & 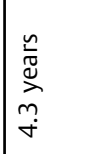 & 1 & 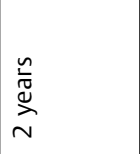 & 1 & 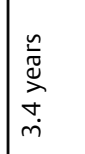 & 1 & 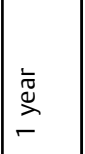 & 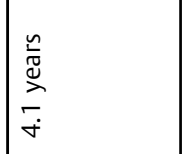 & 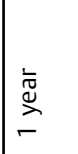 & 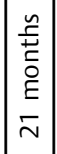 & 1 & 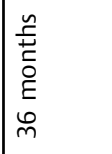 & 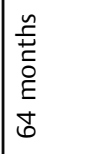 \\
\hline $\begin{array}{l}\text { ô } \\
\text { 文 } \\
\text { 은 }\end{array}$ & 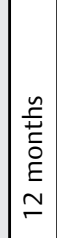 & - & 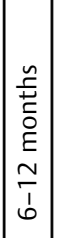 & 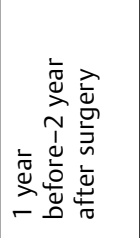 & 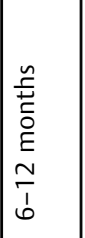 & 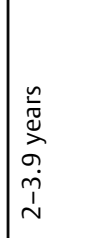 & 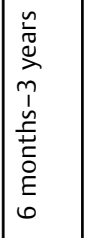 & 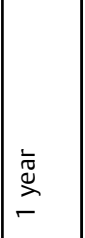 & 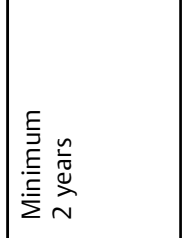 & 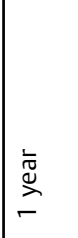 & 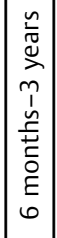 & 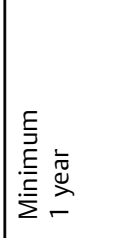 & 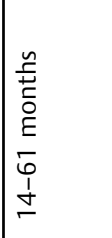 & 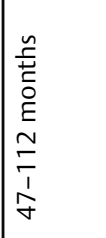 \\
\hline$\frac{\overline{\frac{\sigma}{\sigma}}}{\frac{\pi}{\pi}}$ & $\frac{\tilde{n}}{\tilde{m}}$ & $\frac{\infty}{\stackrel{\infty}{2}}$ & $\frac{n}{\frac{n}{f}}$ & $\frac{\varrho}{\stackrel{\varrho}{f}}$ & 号 & $\frac{\bar{n}}{g}$ & 号 & $\frac{\stackrel{h}{n}}{\stackrel{n}{\sigma}}$ & $\frac{d}{\frac{d}{2}}$ & $\frac{\stackrel{o}{\circ}}{\dot{f}}$ & 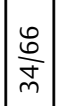 & $\frac{\bar{\sigma}}{9}$ & $\frac{\Gamma}{\infty}$ & 亲 \\
\hline 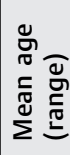 & in & in & i & 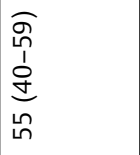 & 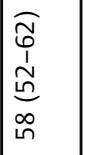 & 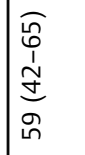 & 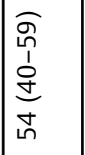 & 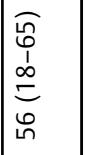 & 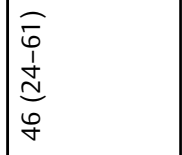 & 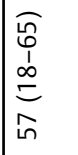 & 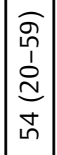 & 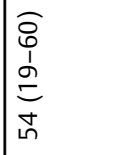 & $\begin{array}{l}0 \\
0 \\
1 \\
o \\
0 \\
m \\
n \\
n\end{array}$ & $\begin{array}{l}0 \\
0 \\
1 \\
\infty \\
0 \\
0 \\
\infty \\
i n\end{array}$ \\
\hline$\frac{0}{0}$ & $\stackrel{0}{\square}$ & ֶู & $\bar{m}$ & ட̊ & 0 & $\stackrel{\stackrel{\infty}{\sim}}{\sim}$ & $\circ$ & in & 吕 & 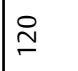 & in & $\overline{8}$ & $\bar{\gamma}$ & 占 \\
\hline 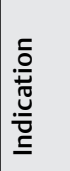 & $\delta$ & The & 1 & $\delta$ & 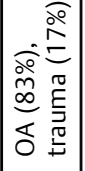 & 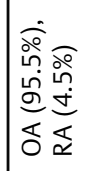 & $\delta$ & 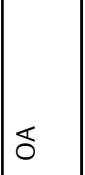 & 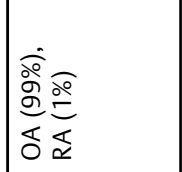 & $\delta$ & రీ & 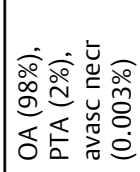 & 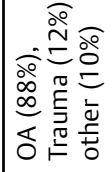 & 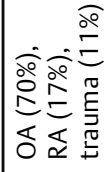 \\
\hline 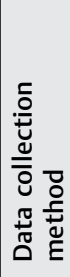 & 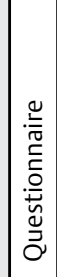 & 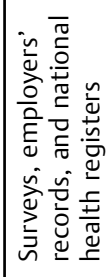 & 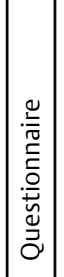 & 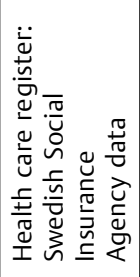 & 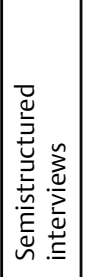 & 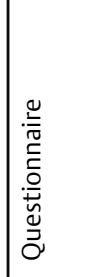 & 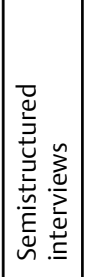 & 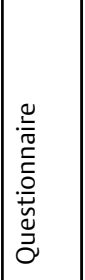 & 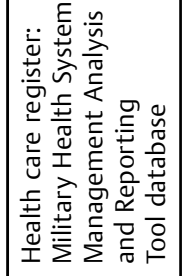 & 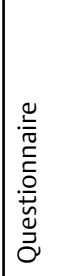 & 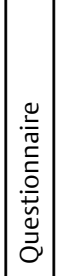 & 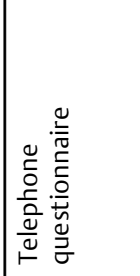 & 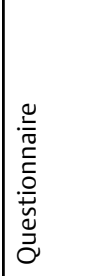 & 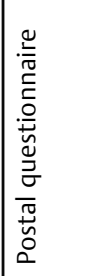 \\
\hline 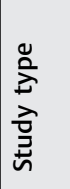 & 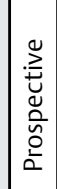 & 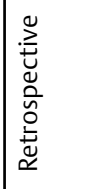 & 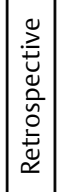 & 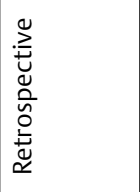 & 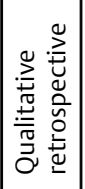 & 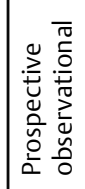 & 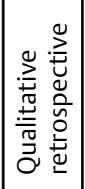 & 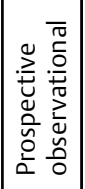 & 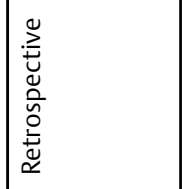 & 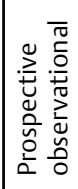 & 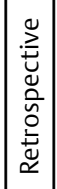 & 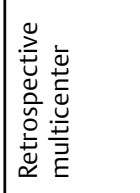 & 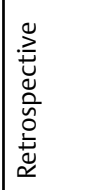 & 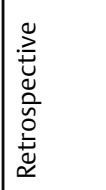 \\
\hline 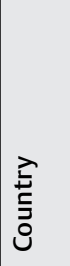 & 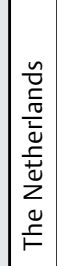 & 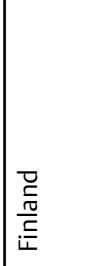 & 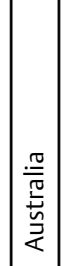 & 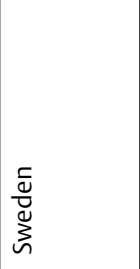 & $\begin{array}{l}\frac{\pi}{0} \\
\stackrel{0}{0} \\
\tilde{J}\end{array}$ & 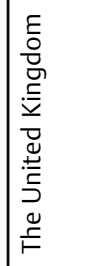 & 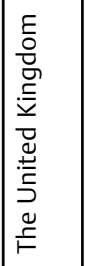 & 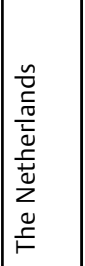 & 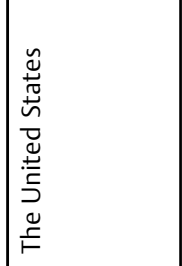 & 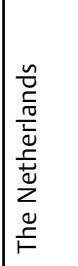 & 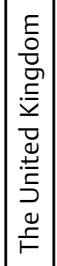 & 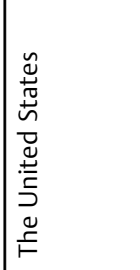 & 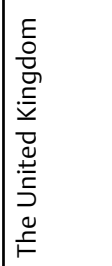 & 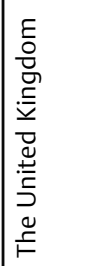 \\
\hline 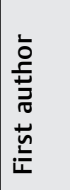 & 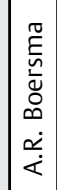 & 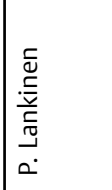 & 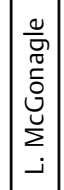 & 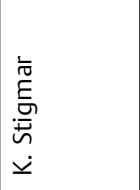 & 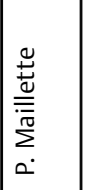 & 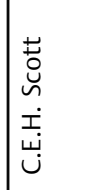 & 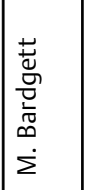 & 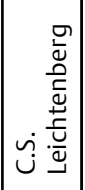 & 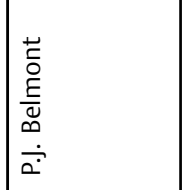 & 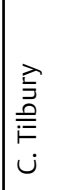 & $\mid$\begin{tabular}{l|}
$\frac{E}{\bar{Q}}$ \\
$\frac{\vec{\omega}}{\dot{a}}$ \\
$\dot{\infty}$ \\
\end{tabular} & 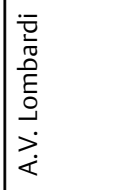 & 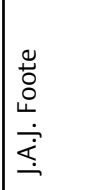 & $\begin{array}{l}\overline{\bar{\pi}} \\
د \\
\dot{I} \\
\dot{I}\end{array}$ \\
\hline 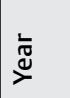 & 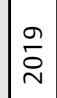 & 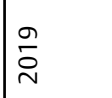 & 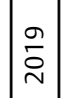 & 㐫 & 产 & $\hat{\stackrel{2}{2}}$ & $\frac{0}{2}$ & $\stackrel{0}{2}$ & $\stackrel{n}{\grave{2}}$ & $\frac{+}{\stackrel{N}{2}}$ & 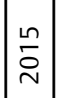 & 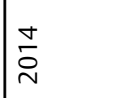 & $\stackrel{\circ}{\stackrel{2}{2}}$ & 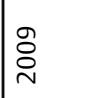 \\
\hline
\end{tabular}




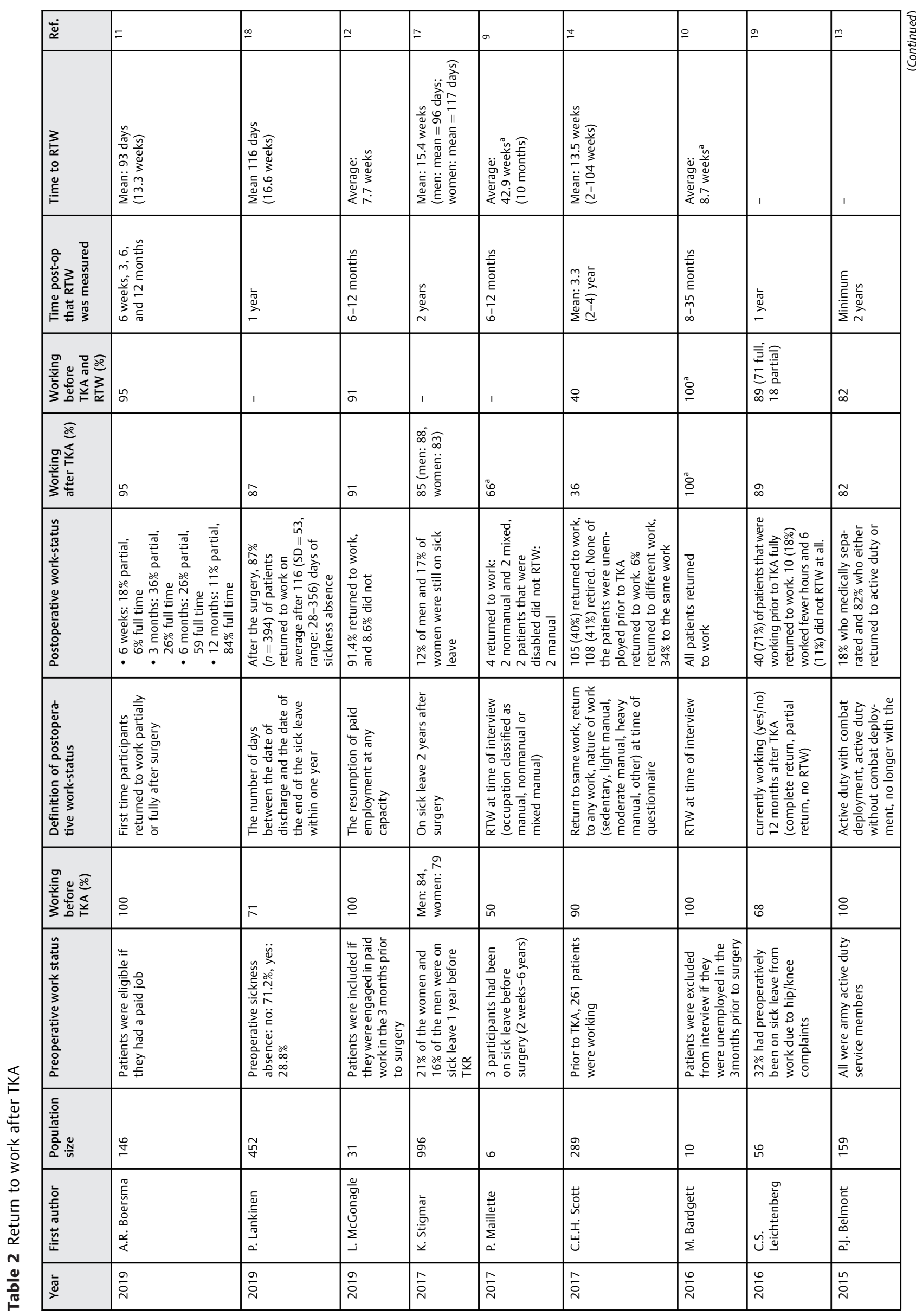




\begin{tabular}{|c|c|c|c|c|c|c|}
\hline$\overleftrightarrow{\ddot{\Xi}}$ & & . & $\bar{\nu}$ & $\cong$ & i & $\cong$ \\
\hline $\begin{array}{l}3 \\
3 \\
\alpha \\
0 \\
0 \\
\tilde{a} \\
\underline{E} \\
1\end{array}$ & & 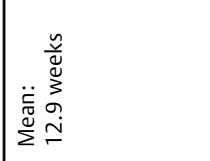 & 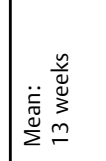 & 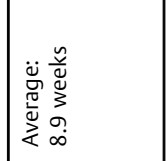 & 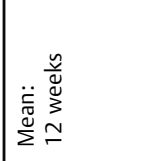 & 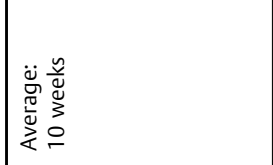 \\
\hline 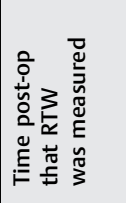 & & 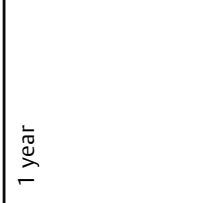 & 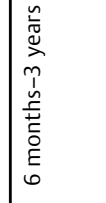 & 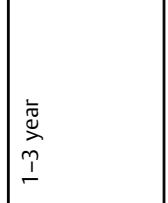 & 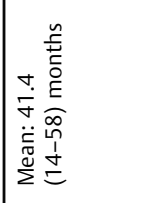 & 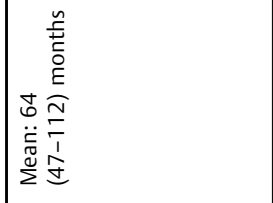 \\
\hline 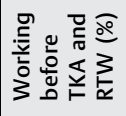 & & $\infty$ & I & $\stackrel{\circ}{\circ}$ & $\infty$ & $\stackrel{\infty}{\infty}$ \\
\hline 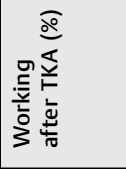 & & f & $\infty$ & $\stackrel{n}{n}$ & in & $\pi$ \\
\hline 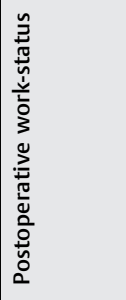 & 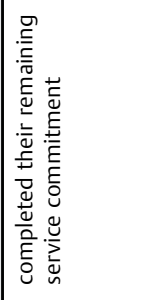 & 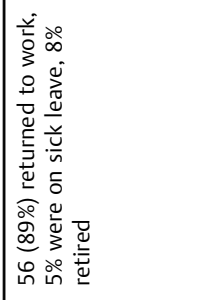 & 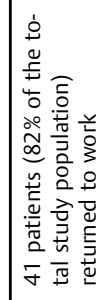 & 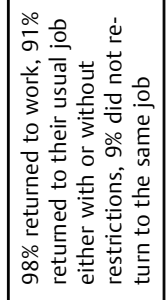 & 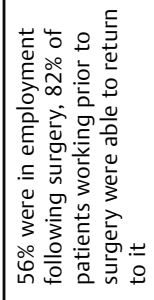 & 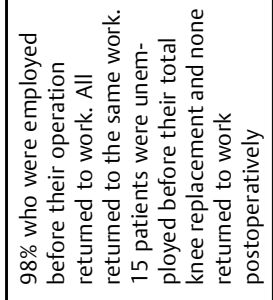 \\
\hline 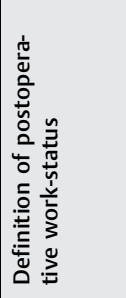 & 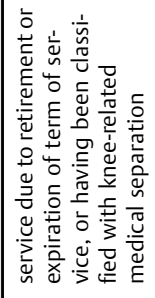 & 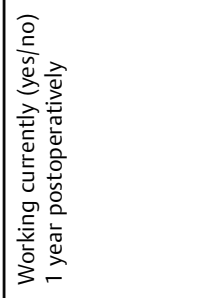 & 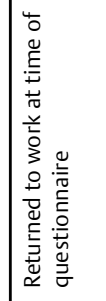 & 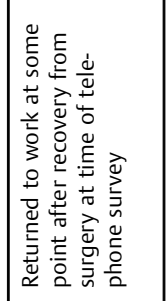 & 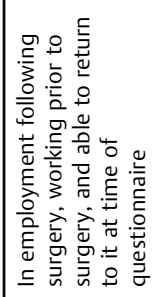 & 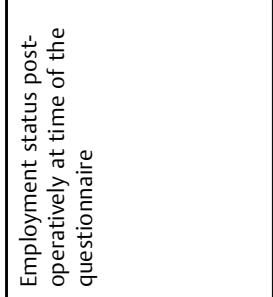 \\
\hline 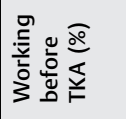 & & $\tilde{n}$ & 亲 & $\stackrel{\text { n }}{\wedge}$ & 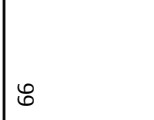 & 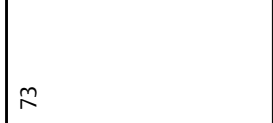 \\
\hline 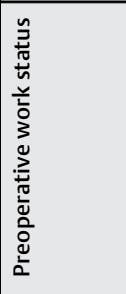 & & 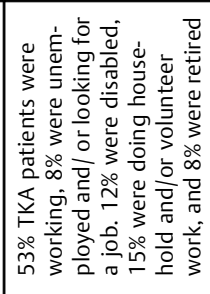 & 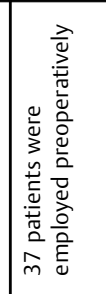 & 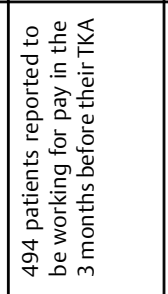 & 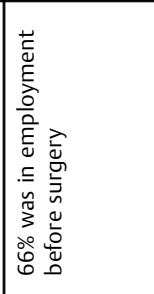 & 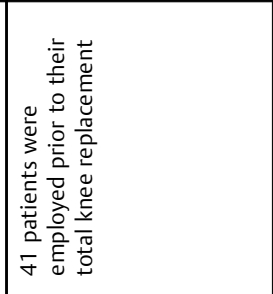 \\
\hline 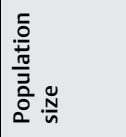 & & 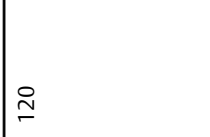 & in & $\bar{\varnothing}$ & F & 号 \\
\hline 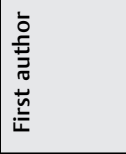 & & 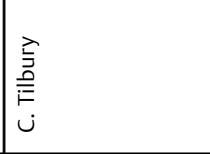 & 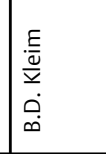 & 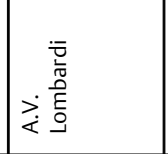 & 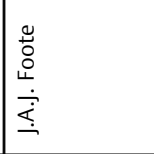 & 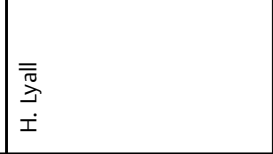 \\
\hline 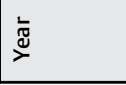 & & $\stackrel{\stackrel{n}{\sigma}}{\stackrel{\alpha}{N}}$ & $\stackrel{n}{\stackrel{n}{N}}$ & $\frac{n}{\grave{n}}$ & $\stackrel{\circ}{\stackrel{0}{2}}$ & @্ণ \\
\hline
\end{tabular}


Table 3 Factors influencing return to work after TKA

\begin{tabular}{|c|c|c|c|c|}
\hline First Author & Associated factors & Limiting of beneficial factors & $\begin{array}{l}\text { Statistical } \\
\text { analysis }\end{array}$ & Ref \\
\hline A.R. Boerma & $\begin{array}{l}\text { In the final model, two covariates } \\
\text { remained: having primarily physically } \\
\text { demanding work (total PA level: } \mathrm{B}=0.499 \text {, } \\
\mathrm{SE}=0.193, p=0.012 \text {; leisure-time PA } \\
\text { level: } \mathrm{B}=0.552, \mathrm{SE}=0.193, p=0.005 \text { ), } \\
\text { and having mixed work tasks (total PA } \\
\text { level: } \mathrm{B}=0.456, \mathrm{SE}=0.171, p=0.009, \\
\text { leisure-time PA level: } \mathrm{B}=0.486, \mathrm{SE} \\
=0.176, p=0.007) \text {. Patients who had a } \\
\text { more physically demanding job or a } \\
\text { combination of a physically and mentally } \\
\text { demanding job needed more time to RTW } \\
\text { than those with a nonphysically } \\
\text { demanding job }\end{array}$ & $\begin{array}{l}\text { Limiting: } \\
\text { - Physically demanding job }{ }^{\mathrm{b}}(p=0.012) \\
\text { - Having mixed work tasks }(p=0.009)\end{array}$ & $\begin{array}{l}\text { Multivariate } \\
\text { analysis }\end{array}$ & 11 \\
\hline P. Lankinen & $\begin{array}{l}\text { Patients with higher non-manual occupa- } \\
\text { tional status had } 2.8(95 \% \mathrm{Cl}: 2.2-3.7 ; \text { in } \\
\text { mutually adjusted model } \mathrm{HR}=2.62 ; \mathrm{Cl} \text { : } \\
1.95-3.52 ; p<0.0001) \text { times higher rate } \\
\text { of RTW as compared with patients with } \\
\text { manual labor occupational status. Low } \\
\text { level of sickness absence ( }<30 \text { days) } \\
\text { before the surgery was associated with a } \\
2.4(95 \% \mathrm{Cl}: 1.9-3.0) \text { times higher rate of } \\
\text { RTW as compared with patients with } \\
\text { longer sickness absence. Those patients } \\
\text { whose self-rated health was good were } 1.4 \\
\text { (95\% Cl: } 1.1-1.7) \text { times more likely to RTW } \\
\text { comparing to those with poor self-rated } \\
\text { general health. Self-rated health was not } \\
\text { associated with RTW in the mutually ad- } \\
\text { justed model }\end{array}$ & $\begin{array}{l}\text { Beneficial: } \\
\text { - Nonmanual work }{ }^{\mathrm{b}}(p<0.0001) \\
\text { - Low number of preoperative sickness } \\
\text { absence days }{ }^{\mathrm{b}}(p<0.0001) \\
\text { - Good self-rated health }(p=0.0012)^{\mathrm{a}}\end{array}$ & $\begin{array}{l}\text { Multivariate } \\
\text { analysis }\end{array}$ & 18 \\
\hline L. McGonagle & $\begin{array}{l}\text { Multiple regression analysis also identified } \\
\text { a significantly earlier time of RTW if flexible } \\
\text { work conditions were resumed } \\
\text { ( } p=0.003 \text { ). Furthermore, those in less } \\
\text { physically demanding jobs (sedentary, } \\
\text { light, and medium) were more likely to } \\
\text { RTW with unchanged conditions of em- } \\
\text { ployment compared with those with more } \\
\text { physically demanding jobs (heavy and very } \\
\text { heavy) ( } 49.5 \text { vs. } 11.8 \% \text { ). Those with more } \\
\text { physically demanding jobs were more } \\
\text { likely to RTW with both reduced hours and } \\
\text { reduced level of duties }\end{array}$ & $\begin{array}{l}\text { Beneficial: } \\
\text { - Flexible work conditions }{ }^{\mathrm{b}}(p=0.003) \\
\text { - Less physically demanding jobs and RTW } \\
\text { with unchanged conditions of } \\
\text { employment }^{\mathrm{b}}\end{array}$ & $\begin{array}{l}\text { Multivariate } \\
\text { analysis }\end{array}$ & 12 \\
\hline K. Stigmar & $\begin{array}{l}\text { Women generally had more sick leave } \\
\text { before surgery and a slower RTW after } \\
\text { both THR and TKR }\end{array}$ & $\begin{array}{l}\text { Limiting: } \\
\text { Women }\end{array}$ & - & 17 \\
\hline P. Maillette & $\begin{array}{l}\text { Participants' representations arose from } \\
\text { expectations of the surgery, representa- } \\
\text { tions of their current condition, and } \\
\text { perceived support from the various envi- } \\
\text { ronmental systems. The patients that did } \\
\text { RTW felt they had experienced greater } \\
\text { improvement after surgery and received } \\
\text { concrete support from their workplace, } \\
\text { which facilitated their RTW in their view }\end{array}$ & $\begin{array}{l}\text { Limiting: } \\
\text { - High expectations of surgery } \\
\text { - Absence of support from various envi- } \\
\text { ronmental systems } \\
\text { Beneficial: } \\
\text { - Support from the workplace }\end{array}$ & - & 9 \\
\hline C.E.H. Scott & $\begin{array}{l}\text { The functional outcome of TKA, as } \\
\text { assessed by PROMs, was significantly } \\
\text { associated with a return to any work on } \\
\text { univariate analysis. Multivariate analysis } \\
\text { found that, in patients aged } \leq 65 \text { years } \\
\text { working prior to TKA, age }(B=0.156, C l \text { : } \\
0.07-0.24 ; p<0.001) \text { and heavy or mod- } \\
\text { erate manual work }(B=0.960, C l \text { : }\end{array}$ & $\begin{array}{l}\text { Limiting: } \\
\text { - Older age }{ }^{\mathrm{b}}(p<0.001) \\
\text { - Physical nature of employment (manual } \\
\text { jobs; } p=0.03) \\
\text { Beneficial: } \\
\text { - Functional outcome of TKA }{ }^{\mathrm{a}}\end{array}$ & $\begin{array}{l}\text { Univariate and } \\
\text { multivariate } \\
\text { analysis }\end{array}$ & 14 \\
\hline
\end{tabular}


Table 3 (Continued)

\begin{tabular}{|c|c|c|c|c|}
\hline First Author & Associated factors & Limiting of beneficial factors & $\begin{array}{l}\text { Statistical } \\
\text { analysis }\end{array}$ & Ref \\
\hline & $\begin{array}{l}0.18-1.74 ; p=0.03 \text { ) independently pre- } \\
\text { dicted both return to any work and return } \\
\text { to the same work }\end{array}$ & & & \\
\hline M. Bardgett & $\begin{array}{l}\text { Three themes were identified that influ- } \\
\text { enced the process of RTW, from the } \\
\text { patient's perspective. These were delays in } \\
\text { surgical intervention, limited and often } \\
\text { inconsistent advice from healthcare pro- } \\
\text { fessionals regarding RTW, and finally the } \\
\text { absence of rehabilitation to optimize } \\
\text { patient's recovery and facilitate RTW }\end{array}$ & $\begin{array}{l}\text { Limiting: } \\
\text { - Delay in surgical intervention } \\
\text { - Limited advice from healthcare } \\
\text { professionals } \\
\text { - Absence of rehabilitation }\end{array}$ & - & 10 \\
\hline $\begin{array}{l}\text { C.H. } \\
\text { Leichtenberg }\end{array}$ & $\begin{array}{l}\text { In TKA patients, the only variable associ- } \\
\text { ated with a full RTW was change in KOOS } \\
\text { Sport subscale score from baseline } \\
(p=0.039) \text {. } \\
\text { Exploratory multivariable stepwise logistic } \\
\text { regression indicated that self-employment } \\
(p=0.019) \text {, preoperative absence from } \\
\text { work ( } p=0.001) \text { and baseline KOOS ADL } \\
\text { subscale scores ( } p=0.272) \text { were associat- } \\
\text { ed with a RTW. } \\
\text { On univariate analysis, patients who did } \\
\text { not or only partially returned to work were } \\
\text { significantly more likely be older } \\
(p=0.010) \text {, have a lower level of education } \\
(p=0.043), \text { be self-employed ( } p=0.019) \\
\text { and have preoperative absence from work } \\
(p=0.001)\end{array}$ & $\begin{array}{l}\text { Limiting: } \\
\text { - Preoperative absence from work }{ }^{\mathrm{b}} \\
(p=0.001) \\
\text { - Being self-employed }{ }^{\mathrm{b}}(p=0.019) \\
\text { - Older age }{ }^{\mathrm{a}} \\
\text { - Lower level of education }{ }^{\mathrm{a}} \\
\text { Beneficial: } \\
\text { - Change in KOOS Sport subscale score } \\
\text { from baseline } \\
\text { - KOOS ADL subscale scores }(p=0.272)\end{array}$ & $\begin{array}{l}\text { Univariate and } \\
\text { multivariate } \\
\text { analysis }\end{array}$ & 19 \\
\hline P.J. Belmont & $\begin{array}{l}\text { Multivariate analysis showed that the }<45 \\
\text { years age group had a significantly in- } \\
\text { creased odds ratios for being medically } \\
\text { separated (OR }=2.36 ; 95 \% \mathrm{Cl}: 1.14-4.90 \text {; } \\
p=0.0206)\end{array}$ & $\begin{array}{l}\text { Limiting: } \\
\bullet<45 \text { years group }{ }^{\mathrm{b}}(p=0.0206)\end{array}$ & $\begin{array}{l}\text { Univariate and } \\
\text { multivariate } \\
\text { analysis }\end{array}$ & 13 \\
\hline C. Tilbury & $\begin{array}{l}\text { Comparison between those who had } \\
\text { returned to work }(n=64 \text { and } n=56) \text { as } \\
\text { compared with those who had not } \\
\text { returned to work after } 1 \text { year and were not } \\
\text { retired ( } n=5 \text { and } n=6) \text {, did not show any } \\
\text { statistically significant differences }\end{array}$ & $\begin{array}{l}\text { No statistically significant differences } \\
\text { shown }\end{array}$ & $\begin{array}{l}\text { Mann-Whitney } \\
\text { U-test, } \\
\text { Chi-squared test }\end{array}$ & 7 \\
\hline B.D. Kleim & $\begin{array}{l}\text { Patients who have preoperative sick leave } \\
\text { due to their hip or knee arthritis take } 4.6 \\
\text { weeks longer to RTW than those who do } \\
\text { not. Patients with level } 2 \text { or } 3 \text { qualifications } \\
\text { returned to work (mean }=9.9 \text { weeks) sig- } \\
\text { nificantly more quickly than patients with } \\
\text { no qualifications or level } 1 \text { (secondary } \\
\text { education) qualifications (mean }=12.6 \\
\text { weeks). Those with further education be- } \\
\text { yond the required secondary education, } \\
\text { represented by levels } 2 \text { and } 3 \text {, returned } \\
\text { more quickly. Patients in occupations with } \\
\text { a manual level of } 0 \text { on average returned to } \\
\text { work } 2.5 \text { weeks faster than those in } \\
\text { occupations with a manual level of } 1 \\
\text { ( } p=0.026 \text { ). Patients in occupations with a } \\
\text { manual level of } 1 \text { returned to work on } \\
\text { average } 6.2 \text { weeks faster than patients in } \\
\text { occupations with a manual level of } 2\end{array}$ & $\begin{array}{l}\text { Limiting: } \\
\text { - Preoperative sick leave } \\
\text { Beneficial: } \\
\text { - Higher education }{ }^{a} \\
\text { - Nonmanual jobs }\end{array}$ & $\begin{array}{l}\text { Univariate analysis, } \\
t \text {-test, Chi-squared } \\
\text { test }\end{array}$ & 21 \\
\hline A.V. Lombardi & $\begin{array}{l}\text { Stratified by preoperative physical demand } \\
\text { category, there was no difference between } \\
\text { groups in either need for restrictions or } \\
\text { length of time worked with postoperative } \\
\text { restrictions }\end{array}$ & $\begin{array}{l}\text { No statistically significant differences } \\
\text { shown for physical demand category }\end{array}$ & Chi-squared test & 15 \\
\hline
\end{tabular}


Table 3 (Continued)

\begin{tabular}{|c|c|c|c|c|}
\hline First Author & Associated factors & Limiting of beneficial factors & $\begin{array}{l}\text { Statistical } \\
\text { analysis }\end{array}$ & Ref \\
\hline J.A.J. Foote & $\begin{array}{l}\text { No statistically significant difference in } \\
\text { physical intensity of pre- versus postoper- } \\
\text { ative occupation was found. } 12 \% \text { found } \\
\text { that their ability to work was significantly } \\
\text { worse, } 37 \% \text { found that their ability to work } \\
\text { was significantly better }\end{array}$ & $\begin{array}{l}\text { No statistically significant differences } \\
\text { shown for physical intensity of occupation }\end{array}$ & $\begin{array}{l}\text { Chi-squared test, } \\
\text { Fisher's exact test }\end{array}$ & 20 \\
\hline H. Lyall & $\begin{array}{l}\text { None of the unemployed patients returned } \\
\text { to work. Of the } 15 \text { patients unemployed } \\
\text { before surgery } 12 \text { had previously undertaken } \\
\text { manual work. } 98 \% \text { of the patients employed } \\
\text { before surgery did RTW }\end{array}$ & $\begin{array}{l}\text { Limiting: } \\
\text { - Being unemployed before surgery } \\
\text { - Manual work } \\
\text { Beneficial: } \\
\text { - Being employed before surgery }\end{array}$ & - & 16 \\
\hline
\end{tabular}

Abbreviations: ADL, activities of daily living; $\mathrm{Cl}$, confidence interval; HR, hazard ratio; OR, odds ratio; KOOS Score, Knee Injury and Osteoarthritis Outcome Score; PA, physical activity; PROM, patient reported outcome measures; Ref., reference; RTW, return to work; SE, standard error; THR, total hip replacement; TKA, total knee arthroplasty; TKR, total knee replacement.

a Statistically significant.

bstatistically significant with multivariate analysis.

$\left(p=0.003^{12}\right)$, older age $\left(p<0.001^{14}\right)$, being self-employed $\left(p=0.019^{19}\right)$, and age less than 45 years in a military population $\left(p=0.0206^{13}\right)$. Factors also shown to be statistically significant were level of education, functional outcome of TKA, change in Knee Injury Osteoarthritis Outcome Score (KOOS) Sport subscale score from baseline, being selfemployed, and a good self-rated health.

The factor most frequently reported was the physical nature of the employment. Six different studies showed that a physically demanding job had a negative impact on RTW of which four studies reported after applying multivariate analysis. Preoperative absence from work was reported by four different studies to be influencing RTW negatively, of which two using multivariate analysis. Factors reported by two different studies were older age, level of education, functional outcome after TKA, and support from the environment and from health care professionals.

\section{Methodological Quality}

-Table 4 summarizes the risk of bias in each domain for all included studies according to the QUIPS tool. Four studies scored low risk of bias in each domain. In eight studies, risk of bias was scored as moderate in one or more domains. In two studies, high risk of bias was found in the "study participation" domain. These last two were qualitative studies using purposive sampling.

\section{Discussion}

This systematic review provides important insights regarding RTW after TKA. Overall, the majority of patients reported RTW

Table 4 Risk of bias according to QUIPS tool

\begin{tabular}{|c|c|c|c|c|c|c|c|}
\hline First author & $\begin{array}{l}\text { Study } \\
\text { participation }\end{array}$ & Study attrition & $\begin{array}{l}\text { Prognostic } \\
\text { factor } \\
\text { measurement }\end{array}$ & $\begin{array}{l}\text { Outcome } \\
\text { measurement }\end{array}$ & $\begin{array}{l}\text { Study } \\
\text { confounding }\end{array}$ & $\begin{array}{l}\text { Statistical } \\
\text { analysis } \\
\text { and reporting }\end{array}$ & Ref. \\
\hline A.R. Boerma & Low bias & Low bias & Low bias & Low bias & Low bias & Low bias & 11 \\
\hline P. Lankinen & Moderate bias & Low bias & Moderate bias & Low bias & Moderate bias & Low bias & 18 \\
\hline L. McGonagle & Moderate bias & Low bias & Moderate bias & Low bias & Moderate bias & Low bias & 12 \\
\hline K. Stigmar & Low bias & Low bias & Low bias & Low bias & Low bias & Low bias & 17 \\
\hline P. Maillette & High bias & Low bias & Moderate bias & Moderate bias & Low bias & Low bias & 9 \\
\hline C.E.H. Scott & Low bias & Moderate bias & Low bias & Low bias & Low bias & Low bias & 14 \\
\hline M. Bardgett & High bias & Moderate bias & Low bias & Low bias & Moderate bias & Moderate bias & 10 \\
\hline C.S. Leichtenberg & Low bias & Moderate bias & Low bias & Low bias & Low bias & Low bias & 19 \\
\hline P.J. Belmont & Low bias & Low bias & Low bias & Low bias & Low bias & Low bias & 13 \\
\hline C. Tilbury & Low bias & Low bias & Low bias & Low bias & Low bias & Low bias & 7 \\
\hline B.D. Kleim & Moderate bias & Moderate bias & Low bias & Low bias & Moderate bias & Moderate bias & 21 \\
\hline A.V. Lombardi & Low bias & Moderate bias & Low bias & Low bias & Low bias & Low bias & 15 \\
\hline J.A.J. Foote & Low bias & Moderate bias & Low bias & Low bias & Moderate bias & Low bias & 20 \\
\hline H. Lyall & Low bias & Moderate bias & Low bias & Low bias & Moderate bias & Moderate bias & 16 \\
\hline
\end{tabular}

Abbreviations: QUIPS, quality in prognosis studies; Ref., reference. 
after TKA but the numbers reported by the included studies were quite divergent.

RTW rate ranged from 40 to $98 \%$ (mean, $89 \%$ ) and the mean time taken to RTW ranged from 7.7 to 16.6 weeks (mean, 13 weeks). In three studies specifying whether patients returned to the same or different jobs, the majority of patients returned to the same job. ${ }^{14-16}$

One study conducted in the United Kingdom by Scott et al reported an RTW rate of $40 \%{ }^{14}$ This is remarkably lower than the other included studies, reporting RTW rates ranging from 71 to $98 \%$. This outlier cannot be explained by the circumstances of the country, as three other studies also conducted in the United Kingdom presented higher RTW rates (82-98\%). ${ }^{16,20,21}$ The majority of patients in this study had a manual job (39\% moderate or heavy manual labor). This was difficult to compare with other studies, as other criteria for physical nature of work were used. However, higher RTW rates were reported in other studies where the majority of patients also had manual jobs (52\% manual jobs and $87 \%$ RTW $){ }^{18}$ Moreover, Scott et al could not find any statistically significant correlation between heavy- or moderate-manual work and $\operatorname{RTW}(p=0.03)$. It also has to be noted that this study has the highest mean age among included studies, and that in this study, the majority of patients not returning to work was aged $>60$ years. However, this age difference compared with other included studies is too small to explain the big difference in RTW rate. Lastly, quality of this study scored good according to the QUIPS tool.

Only two previous systematic reviews on RTW after TKA exist to our knowledge. In 2009, Kuijer et al reported on beneficial and limiting factors affecting RTW in patients undergoing TKA or THA. ${ }^{22}$ They concluded to almost a complete lack of literature on influencing factors on RTW after TKA as only three studies were included in the review of which only one regarding TKA. Later in 2014, Tilbury et al showed that the literature on work status after THA is more extensive than for TKA. ${ }^{7}$ Out of 19 studies included, only 4 provided information on work status after TKA and only 3 were determinants of work status after TKA. They found factors associated with a faster RTW to be female sex, self-employment, higher mental and physical health scores, higher functional comorbidity index scores, and a handicap accessible workspace. Factors associated with a slower RTW were having less pain preoperatively, having a more physically demanding job, and having worker's compensation. These factors were all shown to be significant after multivariate adjustment. However, none of these factors were significantly associated with RTW within 3 months after surgery after multivariate adjustment. ${ }^{23}$ In our study, we also found a physically more demanding job to be associated with a slower or no RTW. Regarding the self-employment and female sex, we found contradictory results. However, in the article included in our review, female sex was not investigated using statistical analysis or multivariate analysis. ${ }^{17}$ Concerning for self-employment, the study included in our review also used multivariate analysis. The authors of this study suggest that this may be related to the observation that self-employed patients generally work more hours than wage earners.
In recent years, there seems to be an increase in interest concerning RTW after TKA. Twelve of the 14 studies included in this review were only recently publicized between 2014 and 2019. Since RTW might have changed over the last years due to rising retirement age, with economic changes and the continuous development of new techniques and prosthesis, the present review can provide valuable new insights in this area.

RTW after major surgery is dependent on various factors such as social and cultural factors, adaptations at the workspace, or the social safety net in the country. These are all factors we could not take into account in this review. We did, however, find no differences in RTW rate between studies conducted in different countries.

It is often unclear whether not returning to work was indeed attributable to the TKA, or if the decision to retire after the operation was already made before surgery. Some patients might postpone the surgery until a time when retirement was possible. However, by limiting the age to 65 years, we partially reduced this bias.

We observed a wide variation in both the definition and timeframe used to measure the work status pre- and postoperatively. Therefore, we did not attempt any statistical analyses as comparisons between studies would be seriously distorted by this heterogeneity. However, efforts were made to minimize the study heterogeneity by applying an upper age limit unlike previous systematic publications. We also obtained a much bigger study population compared with previous reviews, including 14 articles and a total study population of 3,073 patients. To decrease this heterogeneity in future research, standardized uniform outcome measures and protocols for studies investigating RTW after TKA are warranted.

\section{Limitations and Strengths}

We acknowledge several limitations to this study. First of all, we included only articles in English, excluding possibly valuable articles written in any other language. Also, all studies included were conducted in Western countries. Since Eastern countries might differ from Western countries both economically and culturally, conclusions drawn from this review may not be expandable to Eastern countries.

Another weakness to this review is the fact that only 4 of the 14 studies have a prospective design. RTW after a joint replacement is a process with multiple factors involved like retirement age, economic situation and social safety net in the country, surgical techniques, and type of the prosthesis used. A retrospective study is limited by several forms of bias and is therefore not optimal to evaluate the RTW process within its context. More qualitative prospective studies with a sufficient population are therefore warranted.

Furthermore, from the 14 studies included only 6 used multivariate analysis to investigate the correlation between risk factors and RTW. Because of the multitude of factors influencing RTW, adjustment for confounders is essential in these kinds of studies.

At last we encountered a wide variation in study quality, with a risk of bias ranging from low to high. 


\section{Conclusion}

In conclusion, this systematic review found the rate of RTW after TKA to be ranging from 40 to $98 \%$ at a mean of 7.7 to 16.6 weeks. Most important factors associated with a slower or no RTW were a more physical nature of employment and preoperative absence from work.

RTW after TKA is a field of growing interest, with more articles being publicized in the recent years. However, there is still a lack of qualitative large studies applying multivariate analysis. Also, the implication of uniform standardized outcome measures could accommodate a better comparison of studies, benefiting our knowledge regarding this subject. A better understanding of determinants that influence RTW after TKA could after all contribute a more effective and focused management and better preventive measures.

\section{Authors' Contributions}

All authors contributed to the study conception and design. Conceptualization was done by D.V.L. and H.V. Data collection and analysis were performed by D.V.L. and J.N. The first draft of the manuscript was written by D.V.L. Review and editing were done by D.V.L., J.N., H.V., and P.B. All authors commented on previous versions of the manuscript. All authors read and approved the final manuscript.

\section{Funding}

None.

\section{Conflict of Interest}

None declared.

\section{References}

1 Peat G, McCarney R, Croft P. Knee pain and osteoarthritis in older adults: a review of community burden and current use of primary health care. Ann Rheum Dis 2001;60(02):91-97

2 Singh JA, Lewallen DG. Patient-level improvements in pain and activities of daily living after total knee arthroplasty. Rheumatology (Oxford) 2014;53(02):313-320

3 Lange JK, Lee Y-YY, Spiro SK, Haas SB. Satisfaction rates and quality of life changes following total knee arthroplasty in age-differentiated cohorts. J Arthroplasty 2018;33(05):1373-1378

4 Leskinen J, Eskelinen A, Huhtala H, Paavolainen P, Remes V. The incidence of knee arthroplasty for primary osteoarthritis grows rapidly among baby boomers: a population-based study in Finland. Arthritis Rheum 2012;64(02):423-428

5 Kurtz SM, Lau E, Ong K, Zhao K, Kelly M, Bozic KJ. Future young patient demand for primary and revision joint replacement: national projections from 2010 to 2030. Clin Orthop Relat Res 2009;467(10):2606-2612

6 Finnish Centre for Pensions. Retirement ages. Accessed September 10, 2020 at: https://www.etk.fi/en/work-and-pensions-abroad/ international-comparisons/retirement-ages/
7 Tilbury C, Schaasberg W, Plevier JWMJWM, Fiocco M, Nelissen RGHH, Vliet Vlieland TP. Return to work after total hip and knee arthroplasty: a systematic review. Rheumatology (Oxford) 2014; 53(03):512-525

8 Hayden JA, Côté P, Bombardier C. Evaluation of the quality of prognosis studies in systematic reviews. Ann Intern Med 2006; 144(06):427-437

9 Maillette P, Coutu M-FF, Gaudreault N. Workers' perspectives on return to work after total knee arthroplasty. Ann Phys Rehabil Med 2017;60(05):299-305

10 Bardgett M, Lally J, Malviya A, Deehan D. Return to work after knee replacement: a qualitative study of patient experiences. BMJ Open 2016;6(02):e007912

11 Boersma AR, Brouwer S, Koolhaas W, et al. No association between preoperative physical activity level and time to return to work in patients after total hip or knee arthroplasty: a prospective cohort study. PLoS One 2019;14(09):e0221932

12 McGonagle L, Convery-Chan L, DeCruz P, Haebich S, Fick DP, Khan RJK. Factors influencing return to work after hip and knee arthroplasty. J Orthop Traumatol 2019;20(01):9

13 Belmont PJ Jr., Heida K, Keeney JA, Hamilton W, Burks R, Waterman BR. Return to work and functional outcomes following primary total knee arthroplasty in U.S. military servicemembers. J Arthroplasty 2015;30(06):968-972

14 Scott CEH, Turnbull GS, MacDonald D, Breusch SJ. Activity levels and return to work following total knee arthroplasty in patients under 65 years of age. Bone Joint J 2017;99-B(08): 1037-1046

15 Lombardi AV Jr., Nunley RM, Berend KR, et al. Do patients return to work after total knee arthroplasty? Clin Orthop Relat Res 2014; 472(01):138-146

16 Lyall H, Ireland J, El-Zebdeh MY. The effect of total knee replacement on employment in patients under 60 years of age. Ann R Coll Surg Engl 2009;91(05):410-413

17 Stigmar K, Dahlberg LE, Zhou C, Jacobson Lidgren H, Petersson IF, Englund M. Sick leave in Sweden before and after total joint replacement in hip and knee osteoarthritis patients. Acta Orthop 2017;88(02):152-157

18 Lankinen P, Laasik R, Kivimäki M, et al. Are patient-related preoperative factors influencing return to work after total knee arthroplasty. Knee 2019;26(04):853-860

19 Leichtenberg CS, Tilbury C, Kuijer P, et al. Determinants of return to work 12 months after total hip and knee arthroplasty. Ann R Coll Surg Engl 2016;98(06):387-395

20 Foote JAJ, Smith HK, Jonas SC, Greenwood R, Weale AE. Return to work following knee arthroplasty. Knee 2010;17(01): 19-22

21 Kleim BD, Malviya A, Rushton S, Bardgett M, Deehan DJ. Understanding the patient-reported factors determining time taken to return to work after hip and knee arthroplasty. Knee Surg Sports Traumatol Arthrosc 2015;23(12):3646-3652

22 Kuijer PPFM, de Beer MJPM, Houdijk JHP, Frings-Dresen MHW. Beneficial and limiting factors affecting return to work after total knee and hip arthroplasty: a systematic review. J Occup Rehabil 2009;19(04):375-381

23 Styron JF, Barsoum WK, Smyth KA, Singer ME. Preoperative predictors of returning to work following primary total knee arthroplasty. J Bone Joint Surg Am 2011;93(01):2-10 